\title{
Loss of Heterozygosity in the MXI1 Gene Is a Frequent Occurrence in Melanoma
}

Shashi M. Ariyanayagam-Baksh, M.B., B.S., Fabien K. Baksh, M.B., B.S., Patricia A. Swalsky, B.Sc., Sydney D. Finkelstein, M.D.

Department of Pathology, University of Pittsburgh Medical Center, Pittsburgh, Pennsylvania

Melanoma development and progression is thought to be the result of a multi-step accumulation of genetic damage, with loss of heterozygosity in chromosome 9p (MTS1) frequently described. In addition, chromosome 10q allelic loss has been reported, implicating the tumor suppressor gene PTEN/MMAC1 on 10q23.3. The MXI1 gene at 10q24-25 is another candidate tumor suppressor that has only rarely been studied in melanomas, with conflicting results. We used microdissectionbased genotyping to investigate 29 melanomas from 20 patients for loss of heterozygosity in intragenic and flanking microsatellite markers for this latter gene. Concurrently, the MTS1 gene was similarly studied using two flanking microsatellites. Fiftyfour percent (15 of 28) of the informative cases showed loss of heterozygosity for one or both MXI1 markers, as compared with $67 \%$ (16 of 24 ) of the informative cases for MTS1. MXI1 allelic loss was seen more frequently in recurrent/metastatic tumors $(59 \%)$, as compared with in primary $(33 \%)$ lesions. Eighty percent of the primary tumors showed loss of heterozygosity for MTS1, as well as $63 \%$ of recurrent/metastatic ones. We studied more than one tumor in eight patients, with those from three patients showing discordant genetic patterns. One patient showed a metastatic tumor with allelic loss for MXI1 that was not identified in the primary melanoma or a local recurrence. The other two patients showed clonal heterogeneity in MXI1 at synchronous and metachronous metastatic foci. These findings support $M X I 1$ as a putative tumor suppressor gene involved in conventional melanoma progression. Genetic heterogeneity seen in different metastases from the same primary suggests a non-

Copyright (C) 2003 by The United States and Canadian Academy of Pathology, Inc.

VOL. 16, NO. 10, P. 992, 2003 Printed in the U.S.A.

Date of acceptance: June 16, 2003.

Address reprint requests to: Shashi M. Ariyanayagam-Baksh, M.B.,B.S.,

Department of Pathology, Lancaster General Hospital, P.O. Box 3555, 555

North Duke St., Lancaster PA 17604-3555; fax: 717-290-4982; e-mail: smariyan@lancastergeneral.org.

DOI: 10.1097/01.MP.0000087421.44975.1C linear pattern of chromosomal damage, with the development of multiple clones within the primary tumor, each acquiring its own metastatic potential.

KEY WORDS: Clonal diversity, Loss of heterozygosity, Melanoma, MTS1/CDKN2A, MXI1.

Mod Pathol 2003;16(10):992-995

Melanoma is a significant health concern among the Caucasian population and is typically characterized by aggressive biologic behavior, with eventual metastasis and death. Tumorigenesis is thought to be associated with a multistep, temporal accumulation of genetic alterations (1), and in an attempt to understand its biology, the molecular pathogenesis of these lesions is being continuously investigated. Of the chromosomal abnormalities encountered, allelic imbalances of chromosome 9p21, which harbors the MTS1 gene are those frequently described in both sporadic and familial melanomas (2-7). Mutations involving the long arm of chromosome 10 have also been reported in sporadic primary and metastatic melanomas at frequencies ranging from 31 to $58 \%(4,5,8-11)$ and have been associated with a poor prognosis $(4,8)$.

The Max interacting protein (MXI1) is a tumor suppressor gene mapped to chromosome 10q2425. Deletion of genetic material from this site is commonly seen in neural crest-associated tumors such as glioblastomas (12). Rao et al. (11) demonstrated MXI1 loss of heterozygosity (LOH) in $46 \%$ of the primary and metastatic desmoplastic melanomas that they studied, postulating that the embryologic neuroectodermal origin of desmoplastic melanomas may account for this similarity with glioblastomas. Extrapolating on this further, it would not be surprising if conventional melanomas should show similar allelic imbalances in the MXII gene. Herbst et al. (13), however, in their study of 65 sporadic primary and metastatic melanomas, found only 7 to $13 \% \mathrm{LOH}$ for this gene. To our knowledge, these are the only two published studies in the English literature on MXI1 in melanomas. 
We thus sought to further investigate the role of the MXI1 gene in the pathogenesis and progression of conventional melanomas by using a microdissection-based genotyping technique to look for LOH using two microsatellite markers for this gene. We compared these findings to those seen in the MTS1 gene on $9 \mathrm{p} 21$.

\section{MATERIALS AND METHODS}

Seven primary, 2 recurrent, and 20 metastatic melanomas were identified from the files of the Presbyterian and Montefiore University Hospitals of the University of Pittsburgh Medical Center. Paraffin-embedded blocks were obtained for each of the 29 cases, and clinical information was extracted from the Medical Archives Retrieval System.

Serial $4-\mu \mathrm{m}$-thick unstained histologic sections were microdissected under stereoscopic observation, as described elsewhere (14). For each tumor sample, representative uninvolved dermis of approximately equal size was also obtained as a normal control. The microdissected samples were treated with $50 \mu \mathrm{L}$ of proteinase $\mathrm{K}(10 \mathrm{ng} / \mathrm{mL})$ for 2 hours and boiled for 5 minutes to remove enzyme activity.

Each sample was PCR amplified in individual reactions using oligonucleotide primers targeted at microsatellite repeats of the MXI1 and MTS1 genes. For MXI1, we used an intragenic microsatellite (3NT) in the 3' nontranslating region of the gene (12), and the flanking repeat unit D10s1173 (GenBank accession no. L30341). MTS1 was studied using two flanking microsatellites D9s251 and D9s254 (GenBank accession nos. L18726 and L18050, respectively). The primers for MXI1.3NT were 5'-GGTTACTCCAGTGCCAGTGT-3' (sense) and 5'-TTAAATACAGGTCCTCTGACCC-3' (antisense); for D10s1173, they were 5'CATGCCAAGACTGAAACTCC-3' (sense) and 5'AAACCCCAATGCCATAATGG-3' (antisense); for D9s251, they were 5'-CCTGTGTTGAAATTTTGACTG-3' (sense) and 5'-ATTTCAGACTTCCTTGTGTTC-3' (antisense); and for D9s254, they were 5'-TCCTGGGTAATAACTGCCG-3' (sense) and 5'-CACTCACACACACGCTCAG-3' (antisense). After initial denaturation at $95^{\circ}$ for 10 minutes, 35 cycles of PCR were carried out with denaturation at $94^{\circ} \mathrm{C}$ for 1 minute, annealing at $58^{\circ} \mathrm{C}$ for 1 minute, and polymerization at $74^{\circ} \mathrm{C}$ for 1 minute, with a final 10 -minute extension at $74^{\circ} \mathrm{C}$. The amplified products were labeled with $\left[\mathrm{P}^{33}\right]$ deoxyadenine triphosphate and electrophoresed in $6 \%$ polyacrylamide. Loss of heterozygosity was determined by comparing the intensity of the polymorphic bands from normal and tumor tissue. To control for allelic dropout caused by insufficient sample size, the bands from normal tissue were required to be of similar intensity to justify analysis of the tumor samples.

\section{RESULTS}

Twenty patients were identified with 7 primary, 2 recurrent and 20 metastatic melanomas (Table 1). Overall, both genes showed similar degrees of allelic loss, $54 \%$ for MXI1 and $67 \%$ for MTS1. For the MXI1 gene, comparing primary and recurrent/metastatic lesions, 6 and 22, respectively were informative for one or both markers. $\mathrm{LOH}$ was seen in 2 $(33 \%)$ and $13(59 \%)$ of these (Fig. 1). Similarly analyzing MTS1, 5 and 19 of primary and recurrent/ metastatic tumors were informative, with $4(80 \%)$ and $12(63 \%)$ showing $\mathrm{LOH}$, respectively.

More than one tumor was sampled in eight patients. Of these, the pattern of $\mathrm{LOH}$ in both genes was identical in five. One of the discordant cases (Patient 1) was that of a primary melanoma of the left leg that was negative for LOH in MXI1 and recurred 6 months later with a similar result. The metastasis from 1 year later, however, showed acquisition of $\mathrm{LOH}$ in both loci of the MXI1 gene (Fig. 1). MTS1 was not informative. Patient 10 had two distinct metastases sampled within 1 week of each other. The adrenal tumor showed $\mathrm{LOH}$ in all four gene loci studied, whereas the lung was positive in only one locus for each gene (MXI1.3NT and D9s251) and was negative in the other two. The primary tumor, a trunk melanoma from 19 years prior, was not available for analysis. The results from Patient 11 were of particular interest, with the two tumors studied representing neck lymph node and parotid gland metastases that were removed 6 and 8 years, respectively, after a right cheek primary. Although on initial inspection the pattern of gene loss appeared to be the same for both lesions, examination of the allelic bands lost for the MXI1.3NT microsatellite revealed that different ones were deleted in the separate metastases and thus represented genetically distinct clones (Fig. 1).

\section{DISCUSSION}

Genetic alterations involving the long arm of chromosome 10 occur fairly commonly in primary and metastatic melanomas, with frequencies ranging from 31 to $58 \%(4,5,8-11)$. The gene most often implicated is PTEN/MMAC1 $(9,10)$, a tumor suppressor gene located on 10q23.3. Germline mutations of this gene have been associated with Cowden disease (multiple hamartoma syndrome), a rare autosomal-dominant familial cancer syndrome (15). The MXI1 gene is also located on the long arm of chromosome 10 and has been mapped to chromosome 10q24-25. This latter gene acts as a tumor suppressor by negatively regulating the myc oncoprotein. Allelic imbalances have been demonstrated in glioblastomas (12); however, to our knowledge, there have been only two prior studies 


\begin{tabular}{|c|c|c|c|c|c|c|c|c|c|c|c|}
\hline \multirow{2}{*}{ Patient } & \multirow{2}{*}{ Age } & \multirow{2}{*}{ Sex } & \multirow{2}{*}{ Sample Site } & \multirow{2}{*}{ Sample } & \multirow{2}{*}{ TNM } & \multirow{2}{*}{$\begin{array}{l}\text { Breslow Depth } \\
\text { (mm) }\end{array}$} & \multirow{2}{*}{ Clark's Level } & \multicolumn{2}{|c|}{ MXI1 } & \multicolumn{2}{|c|}{ MTS1 } \\
\hline & & & & & & & & $3 \mathrm{NT}$ & D10s1173 & D9s251 & D9s254 \\
\hline \multirow[t]{3}{*}{$1^{a}$} & 57 & $\mathrm{~F}$ & Left leg & $\mathrm{P}$ & ND & ND & ND & - & - & NI & NI \\
\hline & & & Left leg & $\mathrm{R}$ & N/A & N/A & N/A & - & - & NI & NI \\
\hline & & & Groin lymph node & $\mathrm{Me}$ & N/A & N/A & N/A & + & + & NI & NI \\
\hline 2 & 71 & M & Back & $\mathrm{P}$ & pT4aN0Mx & 5.0 & IV & NI & - & NI & NI \\
\hline \multirow[t]{2}{*}{3} & 42 & M & Left back & $\mathrm{P}$ & pT3bN1Mx & 4.0 & IV & + & NI & + & + \\
\hline & & & Left axilla LN & $\mathrm{Me}$ & N/A & N/A & N/A & + & NI & + & + \\
\hline 4 & 63 & M & Right upper arm & $\mathrm{P}$ & pT4aNxMx & 6.5 & IV & NI & NI & NI & + \\
\hline $5^{b}$ & 46 & M & Left waist & $\mathrm{P}$ & pT3aNxMx & 2.68 & IV & - & - & + & + \\
\hline 6 & 28 & M & Scalp & $\mathrm{P}$ & pT3aNxMx & 2.87 & IV & - & - & + & - \\
\hline 7 & 41 & $\mathrm{~F}$ & Left leg & $\mathrm{P}$ & PT2NxMx & 0.49 & III & NI & + & - & - \\
\hline \multirow[t]{2}{*}{8} & 46 & M & Shoulder & $\mathrm{Me}$ & N/A & N/A & N/A & NI & - & + & NI \\
\hline & & & Rib & $\mathrm{Me}$ & N/A & N/A & N/A & NI & - & + & NI \\
\hline 9 & 75 & M & Right cervical LN & $\mathrm{Me}$ & N/A & N/A & N/A & NI & - & - & - \\
\hline \multirow{2}{*}{10} & 59 & M & Left lung & $\mathrm{Me}$ & N/A & N/A & N/A & + & - & + & - \\
\hline & & & Right adrenal & $\mathrm{Me}$ & N/A & N/A & N/A & + & + & + & + \\
\hline \multirow[t]{2}{*}{11} & 44 & M & Right cervical LN & $\mathrm{Me}$ & N/A & N/A & N/A & + & + & - & + \\
\hline & & & Right parotid & $\mathrm{Me}$ & N/A & N/A & N/A & + & + & - & + \\
\hline 12 & 61 & M & Right chest wall & $\mathrm{Me}$ & N/A & N/A & N/A & + & + & NI & - \\
\hline 13 & 35 & M & Left axillary LN & $\mathrm{Me}$ & N/A & N/A & N/A & NI & + & + & NI \\
\hline \multirow[t]{2}{*}{14} & 47 & M & Back & $\mathrm{R}$ & N/A & N/A & N/A & - & - & + & + \\
\hline & & & Right lung & $\mathrm{Me}$ & N/A & N/A & N/A & - & - & + & + \\
\hline 15 & 50 & M & Right adrenal & $\mathrm{Me}$ & N/A & N/A & N/A & - & + & - & NI \\
\hline \multirow[t]{2}{*}{16} & 43 & $\mathrm{M}$ & Right post-auricular LN & $\mathrm{Me}$ & N/A & N/A & N/A & + & NI & - & - \\
\hline & & & Mid-scapula & $\mathrm{Me}$ & N/A & N/A & N/A & + & NI & - & - \\
\hline 17 & 58 & M & Left axillary LN & $\mathrm{Me}$ & N/A & N/A & N/A & NI & + & + & NI \\
\hline \multirow[t]{2}{*}{18} & 73 & $\mathrm{~F}$ & Right adrenal & $\mathrm{Me}$ & N/A & N/A & N/A & NI & - & - & - \\
\hline & & & Lymph node NOS & $\mathrm{Me}$ & N/A & N/A & N/A & NI & - & - & - \\
\hline 19 & 75 & M & Right cervical LN & $\mathrm{Me}$ & N/A & N/A & N/A & NI & - & NI & NI \\
\hline 20 & 39 & M & Right lung & $\mathrm{Me}$ & N/A & N/A & N/A & + & NI & + & + \\
\hline
\end{tabular}

F, female; M, male; P, primary; R, recurrent; Me, metastatic; LN, lymph node; NOS, not otherwise specified; ND, not done; N/A, not applicable; -, no loss of heterozygosity identified; +, loss of heterozygosity identified; NI, sample not informative.

${ }^{a}$ Patient treated with immune therapy prior to excision of primary melanoma, and the specimen was partially submitted for cell culture prior to pathologic evaluation. Accurate pathologic staging thus could not be performed.

${ }^{b}$ Tumor involved the deep excision margin and no subcutaneous tissue was seen. The Breslow depth, Clark's level and TNM stage may thus be greater than reported.

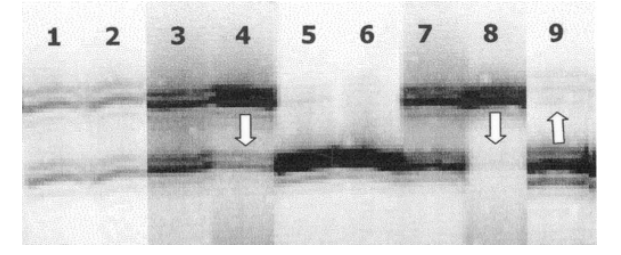

FIGURE 1. MXI1.3NT genotyping of melanomas. Lanes 1 to 4 represent Patient 1 , who is informative for the microsatellite marker, with the specimen from nonlesional dermis showing two bands of similar intensity (Lane 1). Both the primary (Lane 2) and recurrent (Lane 3) melanomas of the left leg show no LOH. The metastasis to the groin lymph node (Lane 4) shows LOH with increased intensity of the upper band and a corresponding decrease of the lower (arrow). Lanes 5 to 6 represent Patient 4, who is noninformative for the gene locus, with only one band present in the normal sample. Lanes 7 to $\mathbf{9}$ depict Patient 11 with a primary melanoma of the right cheek (not shown), with informative normal tissue (Lane 7) and metastases to the parotid gland (Lane 8) and neck lymph node (Lane 9). Mutations involved different allelic bands, confirming that each metastasis is derived from genetically distinct clonal populations.

in the English literature to determine its possible involvement in melanoma tumorigenesis. Herbst et al. (13) concluded that the low frequency of LOH (7 to $13 \%$ ) at intragenic markers for MXI1 in conventional melanomas most likely represented random genetic rather than pathogenic events. Conversely, Rao et al. (11) found MXI1 $\mathrm{LOH}$ to be relatively frequent in desmoplastic melanomas, seen in 5 of 11 informative cases.

Our study, unlike that of Herbst et al. (13), showed frequent $\mathrm{LOH}$ at intragenic and flanking loci of the MXI1 gene, with aberrations seen in $54 \%$ (15 of 28) of the informative cases of primary, recurrent, and metastatic melanomas. This is similar to the $45 \% \mathrm{LOH}$ that was seen in the desmoplastic melanomas studied by Rao et al. (11), although all of our cases were of the conventional type. When the primary melanomas were considered separately, only two of six informative cases (33\%) demonstrated LOH. Conversely, 13 of 22 (59\%) informative metastatic/recurrent lesions showed genetic loss. Although the number of primary lesions studied is small, this seems to suggest that genetic imbalance involving the MXII gene is a late event in tumorigenesis, associated with metastatic potential and spread. Further anecdotal support for this hypothesis is provided by Case 1, in which no $\mathrm{LOH}$ was detected in the primary or recurrent melanoma but there was acquisition of $\mathrm{LOH}$ in the groin metastasis at both the intragenic and flanking loci of the MXI1 gene (Fig. 1).

The findings seen in Patients 10 and 11 are remarkable for their demonstration of genetic heter- 
ogeneity in separate metastases from the same primary. In Patient 10, the adrenal and lung metastases were sampled within 1 week of each other, yet the adrenal tumor showed genetic loss in microsatellites for MXI1 and MTS1 that the lung lesion did not. The synchronous nature of the metastases begs against genetic progression as is seen in Patient 1 but suggests that two distinct clones developed at the same time in the primary tumor and that both acquired metastatic potential. This is further and more convincingly supported by the findings in Patient 11, in which distinct allelic bands were lost in the MXI1 gene in the lymph node and parotid gland metastases (Fig. 1). These findings suggest that the molecular evolution of melanoma is not the result of a uniform, linear progression but involves multiple genetic alterations occurring in clonal subpopulations, each attaining its own metastatic potential. These initial findings support those of others $(6,7)$, but further larger studies need to be performed to investigate the genetic diversity present in melanomas, as well as to correlate these with biologic behavior.

The MTS1 (CDKN2A) gene on chromosome 9p21 has been frequently demonstrated to show allelic loss in both familial and sporadic cutaneous melanomas. This is a tumor suppressor gene that encodes the cell cycle inhibitor $\mathrm{p} 16^{\mathrm{INK} 4 \mathrm{a}}$. In this study, LOH for the MTS1 gene was seen in 16 of 24 informative melanomas $(66.67 \%)$, similar in frequency to previous reports of 47 to $72 \%(2-7,13)$. Of the five informative primary tumors, four $(80 \%)$ showed $\mathrm{LOH}$, also in concordance with other investigators who suggest that mutations involving the short arm of chromosome 9 are early events in the evolution of melanoma (5). The MTS1 analysis was used as a positive control gene in our study and validates our methodology and findings with respect to the much less frequently studied MXI1 gene. The finding of similar degrees of allelic loss in both suggests that MXI1 may also play a role in the development of melanoma, as is believed of MTS1.

In summary, the frequency and pattern of allelic imbalances seen in MXI1 suggest that it is a putative tumor suppressor gene on chromosome 10q involved in conventional melanoma progression. Genetic heterogeneity seen in different metastases from the same primary are indicative of the devel- opment of clonally diverse populations, each acquiring its own metastatic potential.

\section{REFERENCES}

1. Albino AP. Genes involved in melanoma susceptibility and progression. Curr Opin Oncol 1995;7:162-9.

2. Greene MH. The genetics of hereditary melanoma and nevi. Cancer 1999;86:1644-57.

3. Isshiki K, Seng PA, Elder DE, Guerry D, Linnenbach AJ. Chromosome 9 deletion in sporadic and familial melanomas in vivo. Oncogene 1994;9:1649-53.

4. Healy E, Belgaid C, Takata M, Harrison D, Zhu NW, Burd DA, et al. Prognostic significance of allelic losses in primary melanoma. Oncogene 1998;16:2213-8.

5. Healy E, Rehman I, Angus P, Rees JL. Loss of heterozygosity in sporadic primary cutaneous melanoma. Genes Chromosomes Cancer 1995;12:152-6.

6. Morita R, Fujimoto A, Hatta N, Takehara K, Takata M. Comparison of genetic profiles between primary melanomas and their metastases reveals genetic alterations and clonal evolution during progression. J Invest Dermatol 1998;111:11924.

7. Bogdan I, Xin H, Burg G, Boni R. Heterogeneity of allelic deletions within melanoma metastases. Melanoma Res 2001; 11:349-54.

8. Herbst RA, Weiss J, Ehnis A, Cavenee WK, Arden KC. Loss of heterozygosity for 10q22-10qter in malignant melanoma progression. Cancer Res 1994;54:3111-4.

9. Birck A, Ahrenkiel V, Zeuthen J, Hou-Jensen K, Guldberg P. Mutation and allelic loss of PTEN/MMAC1 gene in primary and metastatic melanoma biopsies. J Invest Dermatol 2000; 114:277-80.

10. Reifenberger J, Wolter M, Bostrom J, Buschges R, Schulte $\mathrm{KW}$, Megahed M, et al. Allelic losses on chromosome arm $10 \mathrm{q}$ and mutation of the PTEN (MMAC1) tumour suppressor gene in primary and metastatic malignant melanomas. Virchows Arch 2000;436:487-93.

11. Rao UNM, Bakker A, Swalsky PA, Finkelstein SD. Max interacting protein 1 : loss of heterozygosity is frequent in desmoplastic melanoma. Mod Pathol 1999;12:344-50.

12. Albarosa R, DiDonato S, Finocchiaro G. Redefinition of the coding sequence of the MXI1 gene and identification of a polymorphic repeat in the 3'non-coding region that allows the detection of loss of heterozygosity of chromosome 10q25 in glioblastomas. Hum Genet 1995;95:709-11.

13. Herbst R, Podewski EK, Mommert S, Kapp A, Weiss J. PTEN and MXI1 allelic loss on chromosome 10q is rare in melanoma in vivo. Arch Dermatol Res 1999;291:567-9.

14. Kounelis S, Jones MW, Papadaki H, Bakker A, Swalsky PA, Finkelstein SD. Carcinosarcomas (malignant mixed mullerian tumors) of the female genital tract: comparative molecular analysis of epithelial and mesenchymal components. Hum Pathol 1998;29:82-7.

15. Bussaglia E, Pujol RM, Gil MJ, Marti RM, Tuneu A, Febrer MI, et al. PTEN mutations in eight Spanish families and one Brazilian family with Cowden syndrome. J Invest Dermatol 2002;4:639-44. 